\title{
P04-05. Requirements for antibody domain exchange explored through mutation of the germline $2 \mathrm{GI} 2$ antibody M Huber*, KM Le, KJ Doores and DR Burton
}

Address: Immunology and Microbial Science and IAVI Neutralizing Antibody Center, The Scripps Research Institute, La Jolla, CA, USA

* Corresponding author

from AIDS Vaccine 2009

Paris, France. 19-22 October 2009

Published: 22 October 2009

Retrovirology 2009, 6(Suppl 3):P33 doi:10.1 I86/1742-4690-6-S3-P33

This abstract is available from: http://www.retrovirology.com/content/6/S3/P33

(c) 2009 Huber et al; licensee BioMed Central Ltd.

\section{Background}

2G12 is a high affinity broadly neutralizing monoclonal antibody against a high-mannose glycan cluster on gp120. Its key feature is domain-exchanged heavy chains. Here, we explored the minimal requirements for domainexchange. Although $2 \mathrm{G} 12$ is heavily mutated, only a minority of mutations may be required for domainexchange.

\section{Methods}

Germline 2G12 was derived from the original nucleotide sequence using IMGT/V-QUEST. Germline 2G12 was cloned from genomic DNA and increasing numbers of substitutions corresponding to the wild type 2G12 sequence were introduced in the heavy chain at specific positions, expressed as an IgG in FS293 cells and purified using affinity chromatography. Domain-exchange was assessed by size exclusion chromatography of Fabs prepared by papain digest.

\section{Results}

Germline 2G12 showed neither detectable binding to gp120, nor was the antibody domain-exchanged. If only the light chain was reverted to germline, the antibody retained binding to gp120 and domain-exchange, but not in the heavy chain only chimera.

Increasing numbers of wild type residues, beginning with residues thought to promote domain-exchange (Calarese, Science, 2003, 300: 2065), followed by residues crucial for binding and rare residues in turns, lead to a significantly domain-exchanged antibody.
None of the germline mutants tested detectably bound gp120 or glycans on a standard glycan array.

\section{Conclusion}

Affinity maturation of germline $2 \mathrm{G} 12$ to a domainexchanged antibody requires multiple residues, including framework residues away from the elbow region or $\mathrm{H} / \mathrm{H}^{\prime}$ interface, and is not obligatorily associated with binding to gp120. Further studies will pinpoint the exact requirements for domain-exchange and may provide clues as to how exchange may be promoted through immunization. 